\title{
Fiber Bragg Grating Technology to Construct the Strain Variation Sensing System of an Underwater Pressure Vessel
}

\author{
Kuan-Chieh Chen, ${ }^{1}$ Tso-Sheng Hsieh, ${ }^{1}$ Chao-Wei Wu, ${ }^{2}$ \\ Ching-Yu Hsu, ${ }^{3}$ and Chia-Chin Chiang ${ }^{1 *}$ \\ ${ }^{1}$ Department of Mechanical Engineering, National Kaohsiung University of Science and Technology, \\ 415 Chien Kung Road, Kaohsiung 807, Taiwan, R.O.C. \\ ${ }^{2}$ Department of Aeronautical and Mechanical Engineering, R.O.C. Air Force Academy, \\ No. Sisou 1, Jieshou W. Road, Kaohsiung 820, Taiwan, R.O.C. \\ ${ }^{3}$ Department of Marine Mechanical Engineering, R.O.C. Naval Academy \\ No. 669, Junxiao Rd., Zuoying District, Kaohsiung City 81345, Taiwan, R.O.C.
}

(Received January 18, 2018; accepted June 19, 2018)

Keywords: fiber Bragg grating, pressure sensor, finite element method, underwater pressure vessel

In this study, a fiber Bragg grating (FBG) sensor system was designed and attached to an underwater pressure vessel. The pressure vessel was then subjected to various diving depths of up to $100 \mathrm{~m}$ to determine the sensor system's ability to correctly identify variations in pressure. A total of four FBG sensors were attached to the loader at angles of $0,90,180$, and $270^{\circ}$. The results for each of the four FBG sensors were compared and verified by the finite element method. The experimental results showed that when pressure was imposed on the cylindrical structure, the wavelength variations of the four FBG sensors were 130, 190, 190, and $200 \mu \varepsilon$, respectively. The maximum sensitivity of the FBG pressure sensor is $-22.364 \mu \varepsilon /$ bar. The results indicate that the FBG sensor system can provide accurate measurements of the structural strength of an underwater pressure vessel. In addition, the monitoring technology can be established through comparison and verification with finite element method data. The spectral variations of the proposed optical fiber pressure sensor show good linear relationship with increasing level of strain due to water pressure.

\section{Introduction}

The pressure vessel is the main watertight structural component of an underwater vehicle (UV), which houses the personnel, as well as the marine propulsion system, auxiliary machinery, and sensor systems, in addition to other sensitive equipment. ${ }^{(1)}$ UVs are capable of diving thousands of feet below the water surface, but the safety of a given vessel and its crew can only be ensured through extensive structural testing prior to the vehicle's certification and deployment. Fortunately, as described by Kiddy et al., ${ }^{(2)}$ the accuracy of the finite element method (FEM) for use in such testing and certification has been amply demonstrated. ${ }^{(3)}$

*Corresponding author: e-mail: ccchiang@kuas.edu.tw https://doi.org/10.18494/SAM.2018.1973 
Newly developed UVs will be suitable for deep sea, and they will be tested under deep sea conditions. A diving depth of $6000 \mathrm{~m}$ is desired, which is equal to an ambient pressure of 600 bars. ${ }^{(4)}$ In investigating the pressure hull of novel UVs, Kiddy et al. ${ }^{(5)}$ have employed a FEM to select the placement locations for triaxial, biaxial, and uniaxial fiber Bragg grating (FBG) strain sensors. Subsequently, the given experimental UV was lowered to a depth exceeding 2000 feet, and the optical sensor signals were transmitted via an optical cable to the surface and monitored using a top-side interrogation instrument. The measured temperature-compensated strain values produced by the interrogation instrument demonstrated excellent results. In 2007, Hao et al. ${ }^{(6)}$ conducted a nondestructive evaluation of composite pressure vessels using FBG sensors, but they did not simultaneously verify their experimental results by numerical analysis. An FBG is compact and lightweight, and features multipoint measurement functionality, immediate response, large bandwidth, and high resistance to electromagnetic interference, ${ }^{(7)}$ and can thus serve as a substitute for conventional resistance strain gages to greatly enhance monitoring system efficiency and reliability. In addition, FBG sensors feature an individual diameter of approximately $125 \mu \mathrm{m}$, which is far smaller than their current temperature and flex pressure, and can thus be embedded in various materials, making FBG sensors an indispensable component in the fabrication of intelligent structures. ${ }^{(8,9)}$

In this study, we are concerned with the design of UV pressure vessels using finite element (FE) analysis, and numerical analysis was employed to establish a cylindrical structural model with a T-shaped outer frame. Subsequently, an FBG sensing system was collaboratively employed to measure the strain variations of the model at various depths. Finally, the measurement results were then compared and verified against the results obtained by FEM.

\section{Working Principle of FBG Sensors}

The first in-fiber Bragg grating was demonstrated by Hill et al. ${ }^{(10)}$ in 1978. Fiber grating sensors have since found increasing use in structural applications as deformation monitoring sensors. An FBG relies on the measurement of the reflected light spectrum; therefore, it can also be called a reflection fiber grating. FBG sensors are light, small, and immune to magnetic and electromagnetic interferences, and have good sensitivity, long-term stability, and corrosion resistance. For this study, a simple, low-cost, and fast-responding interrogation system that uses a hybrid FBG-long-period fiber grating arrangement to achieve intensity modulation was developed.

FBGs consist of a section of periodic variation in refractive index in the optical fiber core. When a broadband light spectrum encounters a Bragg grating, a narrow band spectrum at the Bragg wavelength will be reflected; therefore, it can also be called a reflection fiber grating. Each FBG has a grating period of around $1 \mu \mathrm{m}$. When the optocoupler emitted from a broadband light source enters the optical fiber, the light will be reflected because of the change in reflection rate through the FBG. More specifically, the light that meets the conditions of the Bragg grating will be reflected, and the rest of the light will be transmitted. The reflected light rays must have a wavelength that meets the Bragg condition as expressed in the following formula: ${ }^{(11)}$

$$
\lambda_{B}=2 n_{\text {eff }} \Lambda
$$


Here, $\lambda_{B}$ is the Bragg wavelength, $n_{\text {eff }}$ is the effective refractive index, and $\Lambda$ is the grating period. When the grating is under strain loading, wavelength drift can be expressed as

$$
\frac{\Delta \lambda}{\lambda}=\left(1-P_{e}\right) \varepsilon=K_{\varepsilon} \varepsilon
$$

Here, $\varepsilon$ is the strain, $P_{e}$ is the photoelastic constant, and $K_{\varepsilon}$ is the strain wavelength coefficient. According to the literature, ${ }^{(10)}\left(1-P_{e}\right)$ is about 0.78 , and when the grating is subjected to a tensile strain of $1 \mu \varepsilon$, the Bragg wavelength drift will be about $0.0012 \mathrm{~nm}$.

The working principle of the FBG sensor is that the compression and deformation of the cylindrical structure that occur during liquid pressure changes will result in Bragg wavelength shifts. To ensure the UV's functionality, all subsystems are tested in a temperature-controlled pressure tank, which simulates the ambient characteristics of deep sea (10 bars).

\section{Experiments}

A cylindrical pressure vessel with T-shaped frames was fabricated in which the FBG sensor system was adhered. The pressure vessel was made of EH-36 steel and had a diameter of 975 $\mathrm{mm}$ and a thickness of $6.3 \mathrm{~mm}$. Abaqus software was then employed to analyze the strain on the pressure structure at 1 to 10 bar diving depths. The results were then used to analyze static and quasi-static strengths. Subsequently, the FBG was adhered to undergo pressure testing at varying water pressure. The physical test results were then compared with the software simulation results to determine the maximum potential depth at which the UV pressure vessel can safely operate. Figure 1 illustrates the experimental setup of the water pressure test in the static water tank, in which signals from a light source were transmitted to the FBG adhered to the pressure structure. On reaching the FBG, the signals were reflected back along the same optical path and transmitted to a spectrometer, which consequently converted and delivered the signals to a computer for analysis. The FBG signals were scanned through the spectrometer to measure the shift conditions of the characteristic wavelengths and analyze the physical strain endured by the examined object. Observers can use these results to understand the relationship between the wavelength shift and the physical strain exerted, aiding them in employing immediate monitoring actions, as shown in Fig. 2.

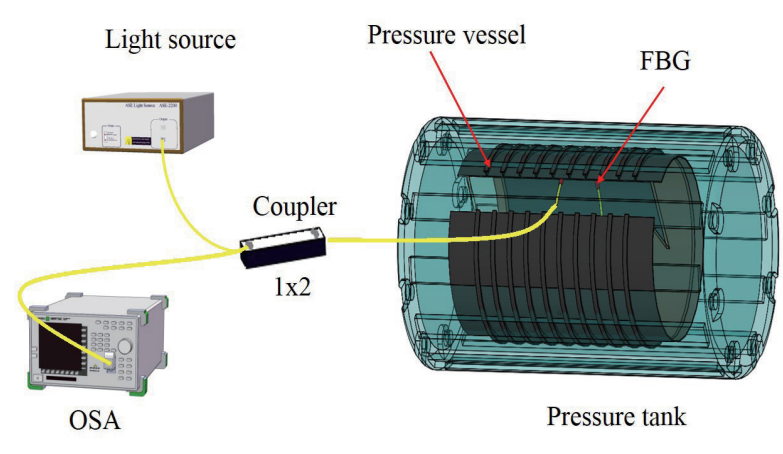

Fig. 1. (Color online) Experimental setup of pressure sensing test.

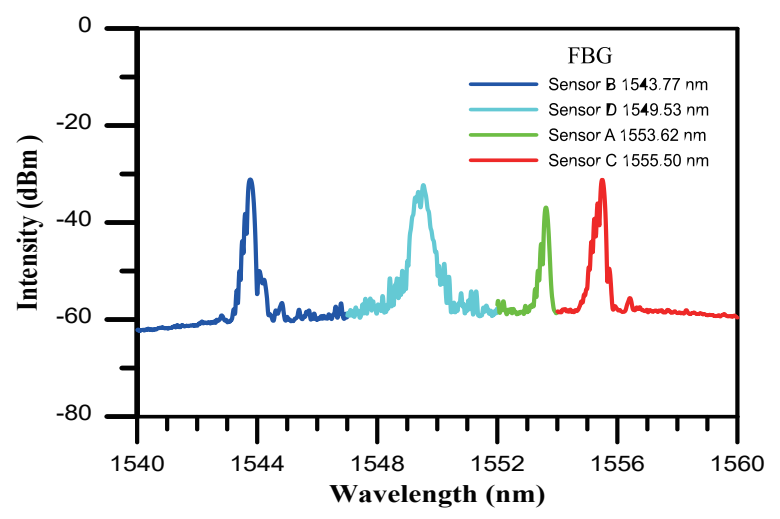

Fig. 2. (Color online) Online monitoring diagram of highly reflective FBG. 


\section{Results and Discussion}

\subsection{Analysis using FEM}

The numerical simulation in this study adopted the Abaqus element serial number S4R, which is a 4-node, 4-sided hyperbolic shell element, to establish the cylindrical pressure vessel. The FEM model is shown in Fig. 3. Each shell element node exhibited six degrees of freedom (ux, uy, uz, $\varphi x, \varphi y$, and $\varphi z$ ), and a degradation integral was established at the curve center of the shell element. For the T-shaped outer frame, the element serial number C3D8R, which is an 8-node, 6-sided solid element construct, was adopted. Subsequently, the structural strength of the pressure structure (T-shaped outer frame) was analyzed. The FEM model comprised a total of 17823 nodes and 24141 elements. The boundary conditions for the cylindrical pressure structure were set so that the front and rear lids were secured using 16 bolts each, and the bolt boundary conditions were fixed. In addition, axial panning and rotation freedom were set at a given constraint. Figure 4 illustrates the static load and deformation strain on the pressure structure at a $100 \mathrm{~m}$ potential depth, indicating that the zones surrounding the T-shaped outer frame were high-stress concentration zones.

\subsection{Pressure testing on framed cylindrical pressure structure}

In this experiment, 1/5 of the model cylindrical pressure vessel was placed in a pressure tank to simulate potential depths of 10 to $100 \mathrm{~m}$. Subsequently, the FBG sensors were placed in four groups (Sensors A, B, C, and D). The cylindrical surface mount device was attached to the pressure vessel to undergo measurements (Fig. 5). Using strain calculation, the maximum strains $\left(100 \mathrm{~m}\right.$ depth) of Sensors A $\left(0^{\circ}\right)$, B $\left(90^{\circ}\right), \mathrm{C}\left(180^{\circ}\right)$, and D $\left(270^{\circ}\right)$ were $130,190,190$, and $200 \mu \varepsilon$, respectively. The sensitivities of Sensors A, B, C, and D were $-15.515,-20.909$, -22.182 , and $-22.364 \mu \varepsilon / \mathrm{bar}$, respectively. Moreover, potential depths were simulated at 10 to $100 \mathrm{~m}$, and the obtained measurement data were then incorporated into the FEM model; the results are illustrated in Fig. 6. The results showed that the measurements for Sensors B, C, and $\mathrm{D}$ were similar to those obtained from the simulation analysis. However, in this experiment, the strain of Sensor A was smaller than those of Sensors B, C, and D because Sensor A was

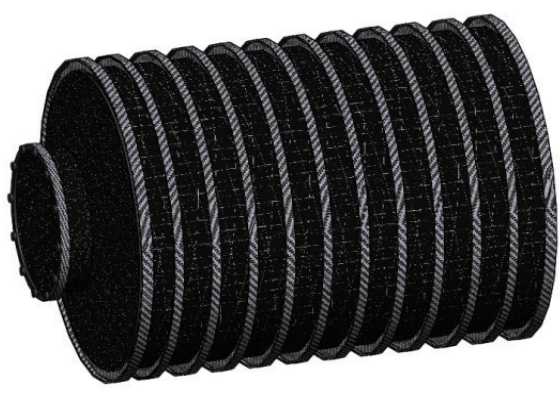

Fig. 3. FEM model.
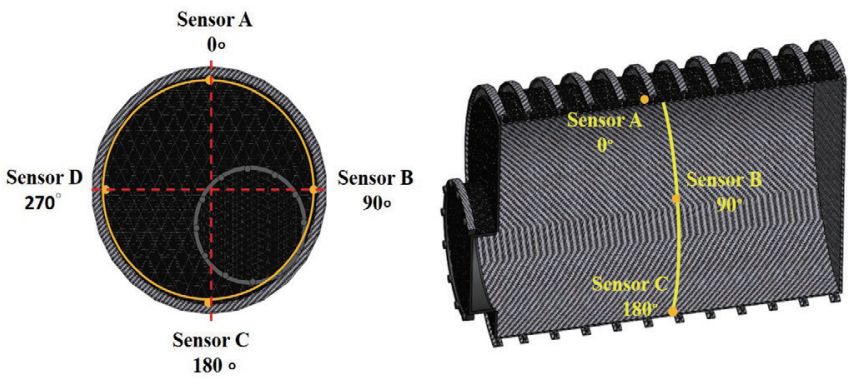

Fig. 4. (Color online) Surface mounting position of FBG sensors. 


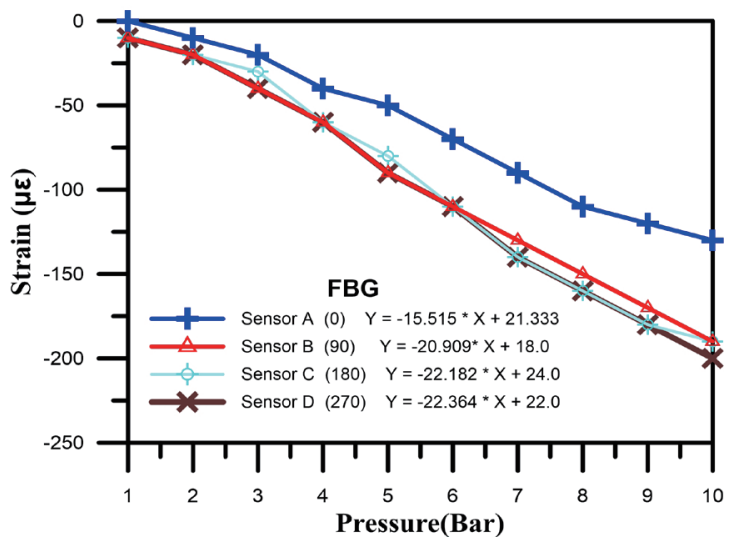

Fig. 5. (Color online) Strain of FBG sensor under 1-10 bar loading.

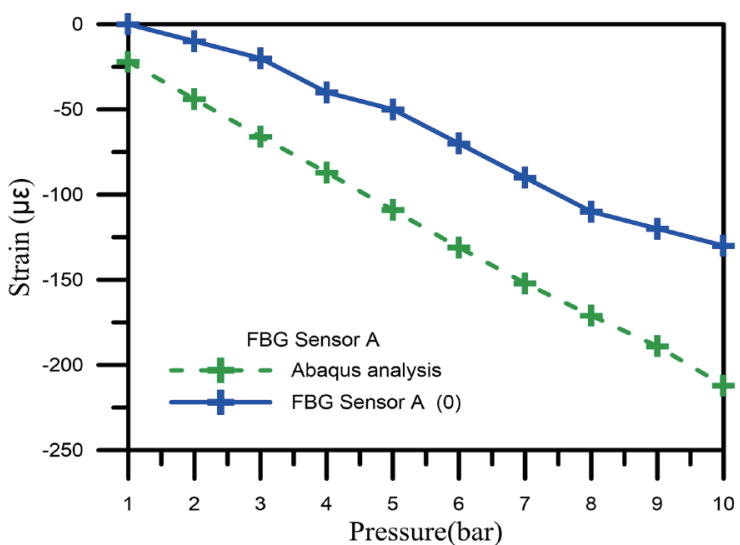

(a)

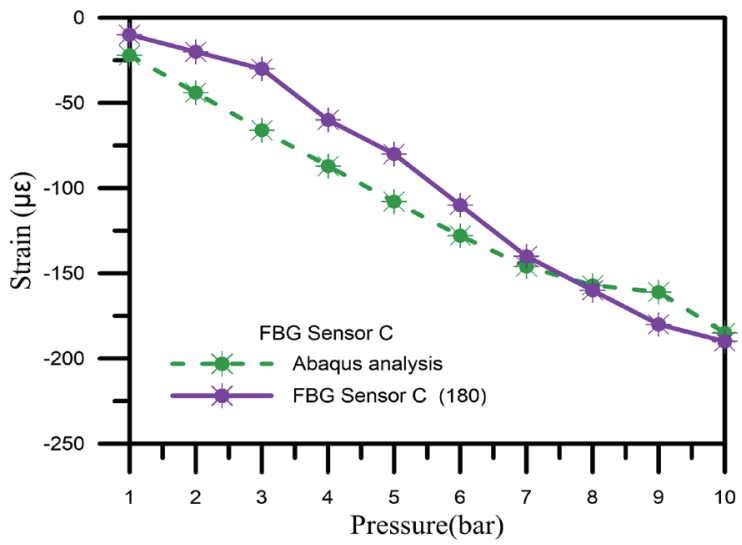

(c)

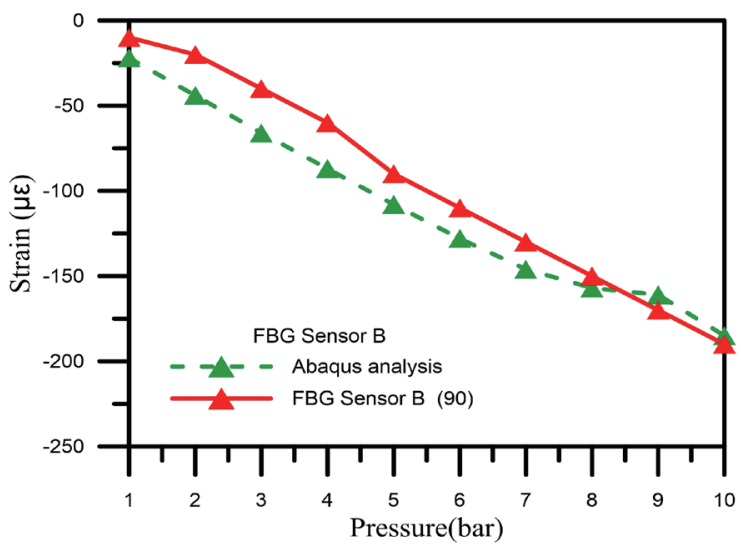

(b)

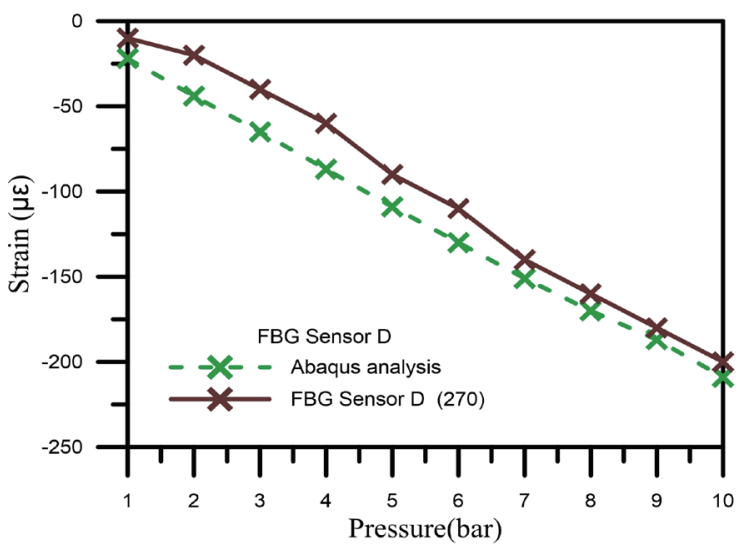

(d)

Fig. 6. (Color online) Comparison of simulation and experimental results of FBG pressure sensor.

attached to the welded site of the cylindrical structure. Figure 6(a) shows that the measurement data is different from the simulation analysis result in Sensor A. This site presented a smaller strain change because the thick reinforcement of the weld causes welded sites to become strong. 


\section{Conclusions}

In this study, we successfully developed an optical fiber grating technique using an excimer laser. In addition, a pressure structure model was established by FEM and then verified by performing actual pressure tests in a pressure tank at up to $100 \mathrm{~m}$. From the stress and strain test results of the cylindrical pressure structure, the wavelength variations of Sensors B, C, and $\mathrm{D}$ were similar to those obtained from the simulation analysis. The maximum sensitivity of the FBG pressure sensor is $-22.364 \mu \varepsilon /$ bar (Sensor D). However, Sensor A was attached to the welded site of the structure, which was subsequently a reinforcement of the weld. Thus, these sites are the locations on a UV that require the most attention during operations. By monitoring the strain deformation of the underwater pressure vessel, the safety of the UV can be increased.

\section{Acknowledgments}

This work was supported by the Ministry of Science and Technology, Taiwan (grant number MOST 107-2623-E-992-301-D).

\section{References}

1 J. R. MacKay and F. V. Keulen: Finite Elem. Anal. Des. 65 (2013) 1.

2 J. S. Kiddy, C. S. Baldwin, and T. J. Salter: Proc. MTS/IEEE Oceans 2 (2005) 1876-1881.

3 B. Mahendra, B. Ankanna, K. T. Reddy, and M. Ravichandra: Int. J. Mod. Eng. Res. 3 (2013) 3552.

4 T. Schmidt, J. Gelze, H. Lehr, and D. Mischnick: OCEANS 2011 (IEEE, 2011) 1.

5 J. S. Kiddy, C. S. Baldwin, and T. J. Salte: Smart Structures and Materials. International Society for Optics and Photonics (2004) 387.

6 J. C. Hao, J. S. Leng, and Z. Wei: Chin. J. Aeronaut. 20 (2007) 120.

7 R. M. Silva, J. M. Baptista, J. L. Santos, A. B. Lobo Ribeiro, F. M. Araújo, L. A. Ferreira, and O. Frazão: Opt. Commun. 291 (2013) 215.

8 W. M. Chen, Wu. J, Zhang. P. L. Liu, and H. Liu: Sens. Lett. 10 (2012) 1476

9 H. P. Gong, X. R. Li, Y. M. Tu, and X. Y. Dong: Sens. Lett. 10 (2012) 1550.

10 K. O. Hill, Y. Fujii, D. C. Johnson, and B. S. Kawasaki: Appl. Phys. Lett. 32 (1978) 647.

11 M. Majumder, T. K. Gangopadhyay, A. K. Chakraborty, K. Dasgupta, and D. K. Bhattacharya: Sens. Actuators, A 147 (2008) 150.

\section{About the Authors}

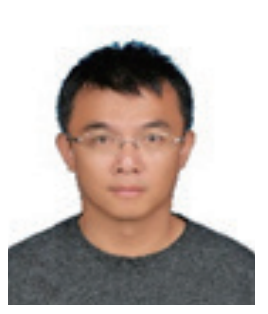

Kuan-Chieh Chen received his B.S. degree from the Department of Naval Architecture and Marine Engineering of Chung Cheng Institute of Technology, National Defense University, Taiwan, in 1999 and his M.S. degree from the Department of Mechanical Engineering of Kun Shan University, Tainan, Taiwan, in 2012. He subsequently began lecturing in the Department of Mechanical Engineering of Kaohsiung University of Applied Sciences, Kaohsiung, Taiwan and became a Ph.D. student in 2014. His research interests include optical fiber sensors and structure analysis. 


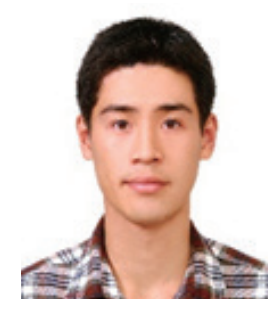

Tso-Sheng Hsieh received his B.S. degree from the Department of Naval Architecture and Marine Engineering of Chung Cheng Institute of Technology, National Defense University, Taiwan, in 1991 and his M.S. degree from the Department of Mechanical Engineering of Kun Shan University, Tainan, Taiwan, in 2007. He received his Ph.D. degree from the Department of Mechanical Engineering of Kaohsiung University of Applied Sciences, Taiwan, in 2016. He subsequently began lecturing in the Department of Industrial Engineering and Management, Fortune Institute of Technology, Kaohsiung, Taiwan and became an assistant professor in 2017. His research interests include optical fiber sensors and structure analysis.

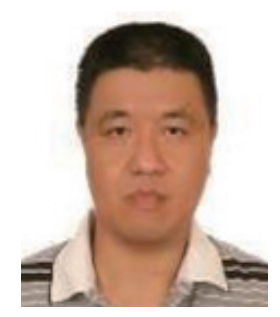

Chao-Wei Wu received his B.S. degree from the Department of Aeronautics and Mechanical Engineering of the R.O.C. Air Force Academy, Taiwan, in 2006 and his M.S. degree from the Department of Aeronautics and Astronautics of Cheng Kung University, Tainan, Taiwan, in 2011. He received his Ph.D. degree from the Department of Mechanical Engineering of Kaohsiung University of Applied Sciences, Taiwan, in 2017. He subsequently began lecturing in the Department of Aeronautical and Mechanical Engineering, Air Force Academy, Kaohsiung, Taiwan and became an assistant professor in 2017. His research interests include optical fiber sensors and micro-electromechanical systems (MEMS).

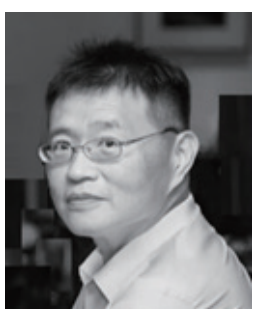

Ching-Yu Hsu received his B.S. and M.S. degrees from the Department of Naval Architecture and Marine Engineering of Chung Cheng Institute of Technology, National Defense University, Taiwan, in 1987 and 1993, respectively, and his Ph.D. degree from Chung Cheng Institute of Technology National Defense University, Taiwan, in 1999. He subsequently began lecturing in the Department of Marine Mechanical Engineering at the R.O.C. Naval Academic, Taiwan and became an associate professor in 2014. His research interests include structure design, underwater shock, and impact simulation and analysis of ships and submarines.

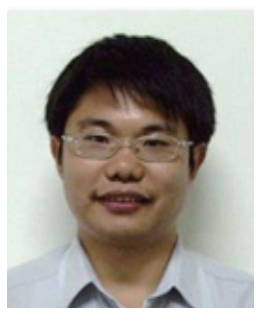

Chia-Chin Chiang received his B.S. and M.S. degrees from the Department of Mechanical and Mechatronic Engineering of National Taiwan Ocean University, Taiwan, in 1998 and 2000, respectively, and his Ph.D. degree from National Taiwan University, Taiwan, in 2005. He subsequently began lecturing in the Department of Mechanical Engineering of Kaohsiung University of Applied Sciences, Taiwan, and became a professor in 2015. His research interests include fiber Bragg gratings (FBGs), optical fiber sensors, and smart materials and structures. 